\title{
Nonsteroidal anti-inflammatory drug (NSAID) associated fixed drug eruption (FDE) in children attending dermatology OPD of a tertiary care hospital of Eastern India: a cross-sectional observational study
}

\author{
Ranjita Santra $^{1}$, Shirsendu Mondal ${ }^{2}$, Patralekha Raychaudhuri ${ }^{3}$
}

\begin{abstract}
${ }^{1}$ Department of Clinical and
Experimental Pharmacology, Calcutta School of Tropical Medicine, Kolkata-700012, West Bengal, India, ${ }^{2}$ Department of Pharmacology, Medical College, Kolkata700012, West Bengal, India, ${ }^{3}$ Department of Pharmacology, Calcutta National Medical College, Kolkata-700014, West Bengal, India
\end{abstract}

Received: 28 July 2013

Revised: 16 January 2014

Accepted: 17 January 2014

*Correspondence to:

Dr. Ranjita Santra,

Email: drvip79@yahoo.co.in

(C) 2014 Santra R et al. This is an open-access article distributed under the terms of the Creative Commons Attribution NonCommercial License, which permits unrestricted noncommercial use, distribution, and reproduction in any medium, provided the original work is properly cited.

\begin{abstract}
Background: Although extensively studied in adults, Nonsteroidal antiinflammatory drug (NSAID) hypersensitivity in children, especially in young children, remains a poorly defined area in both its clinical and epidemiologic aspects.
\end{abstract}

Methods: The present observational study was conducted in the department of Dermatology of a tertiary care hospital in Eastern India. Twenty children (20) were screened with suspected NSAID-associated fixed drug eruption (FDE) in the outpatient department. A thorough history taking and clinical examination was performed for each of the cases of FDE. These cases were then managed conservatively after discontinuation of the suspected medication. Rechallenge with the putative offending drug was not done due to ethical reasons. WHO-UMC Causality Assessment criteria and Naranjo probability scale were used for causality assessment of each of the cases of FDE. The severity of reported reactions was assessed by using Modified Hartwig and Siegel Scale and Preventability of the ADRs was assessed by Modified Schumock and Thornton Scale.

Results: Patients aged between 5 to 12 years and with a male preponderance of 3:2. The offending NSAID was ibuprofen for 8 of the patients, paracetamol and diclofenac for 4 each and ketorolac for 4 of the patients. These patients were prescribed the offending drugs for fever, rheumatoid arthritis and minor trauma. For each patient, history and clinical signs was consistent with the diagnosis of drug-induced FDEs. Causality assessment for each of the cases revealed 'possible' association predominantly (80\%). Severity of the suspected ADR (adverse drug reaction) assessed using Modified Hartwig and Siegel Scale, revealed that the ADRs were mild(30\%) to moderate $(70 \%)$ in severity and of 'probable' preventibility (90\%).

Conclusions: 20 new cases of NSAID-induced FDEs over a period of 6 months suggest that this is not a rare entity as was presumed. There is a growing need for a strict monitoring of such off label offending drugs, known to cause ADRs especially among pediatric patients to ensure safe and rational therapeutics.

Keywords: Nonsteroidal anti-inflammatory drug, Fixed drug eruptions, Children, Causality assessment, Eastern India

\section{INTRODUCTION}

The various side-effects of the drugs have been recorded since time immemorial. They may be trivial or may cause considerable morbidity or mortality. The skin is the largest organ of the body and displays various facets of drug eruption; fixed drug eruption (FDE) is one of them. It is a fascinating adverse drug effect. The safety of drugs administered to pediatric patients is of great concern in the field of therapeutics and demands constant monitoring for adverse events because of altered pharmacokinetics. Fixed drug eruption accounts for about $11 \%-30 \%$ of all adverse drug reactions. ${ }^{1}$ FDE is a distinctive drug-induced dermatosis with a characteristic recurrence at the same sites of the skin or mucous membrane after repeated administration of the causative drug. ${ }^{2}$ It was first described by Bourns in 1889; five years later, it was termed by Brocq as "eruption erythematopigmentee fixee". ${ }^{3}$ The hallmark of this reaction is the occurrence of eruptions in the same location during each 
episode, with additional lesions appearing upon drug rechallenge. Typical locations for the lesions include the genitals, face, hands and feet. Although fixed drug eruption is frequently confused with erythema multiform, the histopathologies are different. Common causes of fixed drug eruption include sulphonamide drugs, tetracycline and nonsteroidal anti -inflammatory drugs.

Nonsteroidal anti-inflammatory drugs (NSAIDs) possess antipyretic, analgesic and anti-inflammatory effects and are frequently used in children for numerous therapeutic indications, the most common ones being fever, minor trauma, postoperative pain and inflammatory disorders, such as juvenile idiopathic arthritis (JIA) and Kawasaki disease. Acetylsalicylic acid (ASA) and other nonsteroidal anti-inflammatory drugs (NSAIDs) are a group of medications with heterogenic chemical structures, sharing the capability of inhibiting with various degrees of specificity and efficacy the cyclooxygenase (COX) enzymes responsible for the prostaglandin synthetase pathway of arachidonic acid metabolism. This blockade also results in the shunting of arachidonic acid toward the 5-lipoxigenase pathway, resulting in increased production and release of cysteinyl leukotrienes. ${ }^{4}$

This article attempts to provide a close insight into the incidence of fixed drug eruptions that occur in the pediatric age group yet remain unreported. It is widely felt that active drug surveillance is of utmost significance in order to capture risk information of drugs being used in children. With this goal in mind, the present study depicts the reported cases of NSAID induced fixed drug eruptions (FDEs) in pediatric patients. The present work focuses on the FDEs affecting the skin and mucous membrane predominantly that were reported in pediatric patients who were on NSAID therapy for various indications such as fever, trauma or rheumatoid arthritis at this hospital. All signs, except scarring due to cutaneous eruptions resolve with the discontinuation of therapy, so early recognition are important.

\section{METHODS}

The study was of cross-sectional observational type in design, which was carried out in the outpatient department of Dermatology during the year 2012 tertiary care government hospital of Eastern India. The study commenced after due approval from the Institution Human Ethics Committee. A total of 20 (twenty) cases in the age group of 5-12 years of fixed drug eruptions were reported and documented within a period of six months. In every case, a detailed history was elicited and a thorough clinical examination was carried out as suggested by Sacerdots et al. ${ }^{5}$ To establish the etiologic agent for a particular type of reaction, attention was paid to the medication history, temporal correlation with the intake of the suspected drug, duration of the rash, approximate incubation period, morphology of the eruption, associated mucosal or systemic involvement and improvement of lesions on withdrawal of the suspected drug. If more than one drug was thought to be responsible, the most likely offending agent was noted and the impression was confirmed by subsidence of the rash on withdrawal of the suspected drug. The rashes were attributed to a drug following the guidelines of Boston collaborative drug reaction surveillance programme. ${ }^{6}$ All the information was carefully recorded in a specially designed proforma. Withdrawal of the suspected NSAID provided a gradual subsidence of the clinical features for each of the children diagnosed with FDEs. Conservative treatment was done for the associated symptoms. The doses of the drugs being prescribed were within the recommended therapeutic dose range for specific indications. Rechallenge with the putative offending drug was not done due to ethical reasons.

\section{Causality assessment}

The causality relationship between suspected drug and reaction was established by using WHO-UMC Causality Assessment criteria and Naranjo probability scale. The causality of reported reactions was categorized to any one of the following categories based on the scale used:

WHO assessment Scale: ${ }^{7}$ Certain, probable, possible, unassessable/unclassifiable, unlikely, conditional/unclassified.

Naranjo's Algorithmic Scale: ${ }^{8}$ Definite, probable and possible;

\section{Assessment of severity}

The severity of reported reactions was assessed by using Modified Hartwig and Siegel Scale ${ }^{9}$ and was categorized into mild, moderate and severe.

\section{Assessment of preventability}

Preventability of the ADRs was assessed by Modified Schumock and Thornton Scale. ${ }^{10}$ This scale of preventability classifies the ADRs as definitely preventable, probably preventable, and not preventable.

\section{RESULTS}

A total of 20 (twenty) patients of pediatric age-group with diagnosed fixed drug eruptions (FDEs) were observed during the study period. There were 12 (twelve) male children and 8 (eight) female children, with an age range of 5-12 years (mean age $=8.5$ years). The incubation period for maculopapular rash and urticaria varied from 30 minutes to 3 weeks in all the cases. The offending NSAIDs were ibuprofen for $8(40 \%)$ of the patients, paracetamol and diclofenac for 4 each $(20 \%+20 \%)$ and ketorolac for $4(20 \%)$ of the patients as shown in the diagram (Figure 1). 


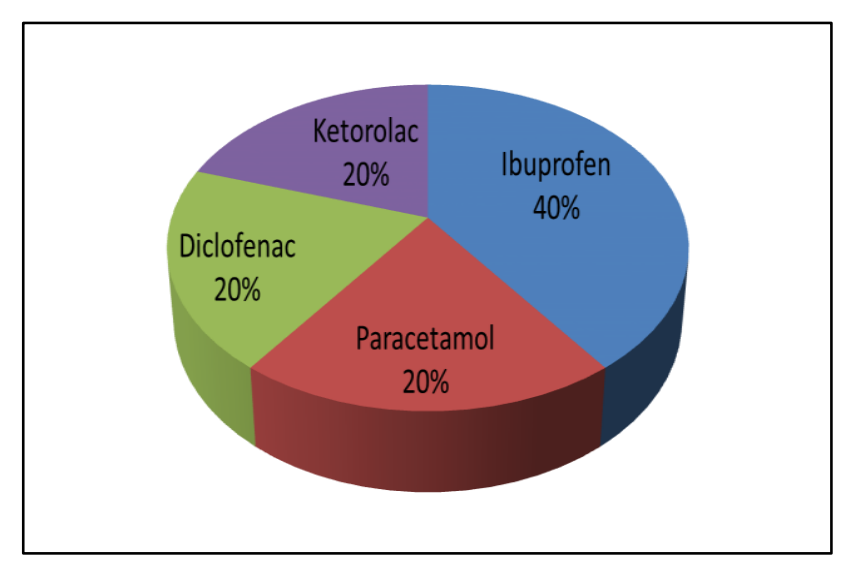

Figure 1: Piechart showing the relative percentage of the NSAIDs associated with FDEs in pediatric agegroup.
Fixed drug eruption (FDE) had an incubation period ranging from two days to two months. None of our patients were rechallenged with the putative offending drug. However, prolonged avoidance has provided a sustained remission from symptoms in all the 20 patients. Causality Assessment was carried out accordingly. Causality assessment for each of the cases of FDE revealed 'possible' association (80\%) predominantly, using WHO-UMC criteria and Naranjo's scale. Severity of the suspected ADR (adverse drug reaction) assessed using Modified Hartwig and Siegel Scale, revealed that ADRs were mild $(30 \%)$ to moderate $(70 \%)$ in severity. Assessment by Modified Schumock and Thornton Scale of ADR preventability showed that most of the ADRs had a score of 'probable' preventability (90\%) among the pediatric patients. A relatively lower number (9\%) of ADRs were not preventable as shown in Figure 2.

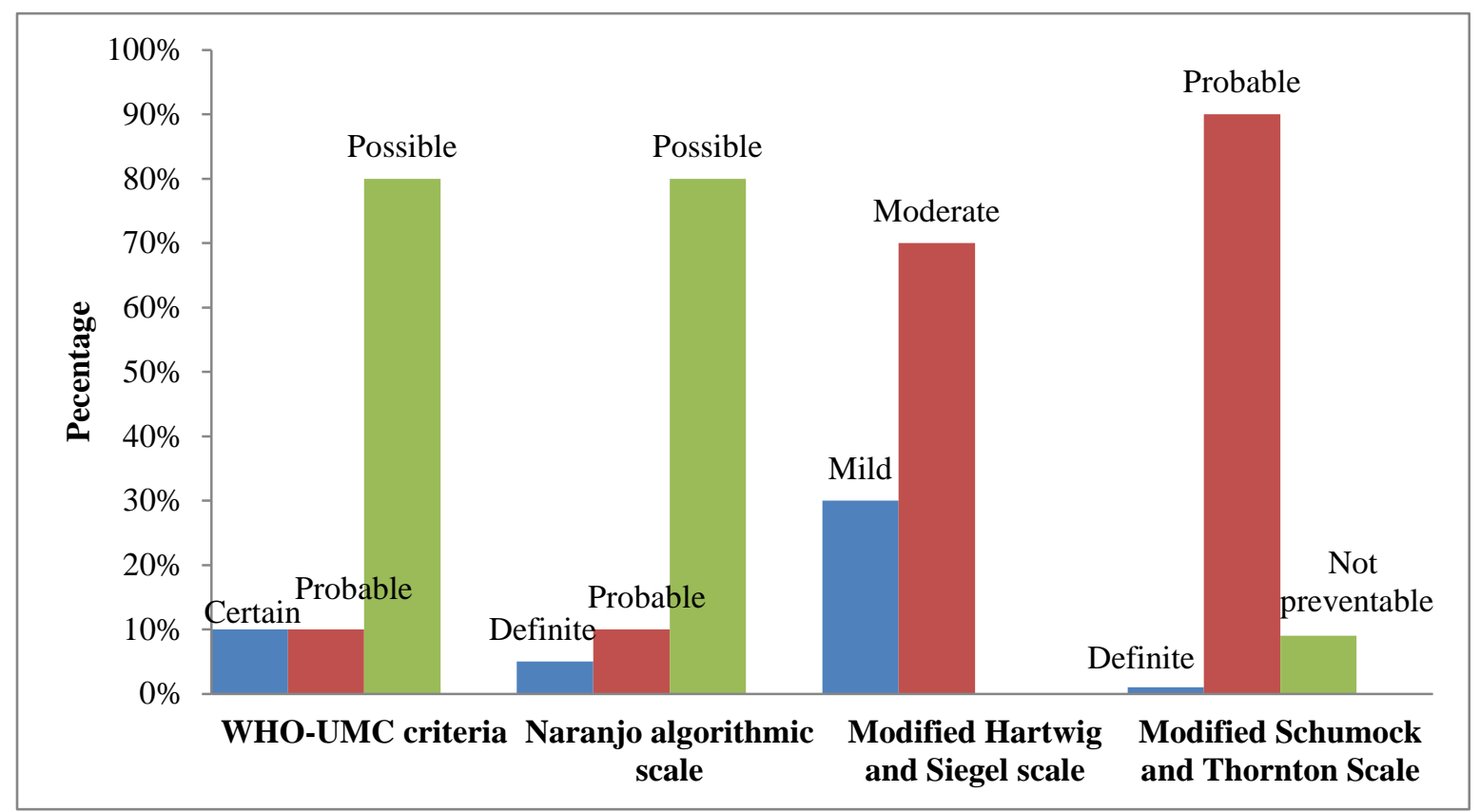

Figure 2: Causality, severity, and preventability of adverse drug reactions (ADRs) associated with NSAIDs in pediatric age-group.

\section{DISCUSSION}

Causality assessment revealed 'possible' association in majority of the cases with the NSAID of a particular chemical class used for specific indications in children, using WHO-UMC criteria and Naranjo's probability scale. Severity of the suspected ADR assessed using Modified Hartwig and Siegel Scale, revealed that ADRs were mild to moderate in severity and were probably preventable as was assessed using Modified Schumock and Thornton Scale. Ibuprofen accounted for maximum number of the cases followed by paracetamol, diclofenac and ketorolac, which is consistent with an earlier study conducted in a tertiary care teaching hospital of South
India. ${ }^{11}$ We observed male preponderance in this study as already been reported earlier in another study. ${ }^{12}$ Earlier maximum number of cases were seen in the $3^{\text {rd }}$ and $4^{\text {th }}$ decade, which is not in conformity with this study. ${ }^{13}$ However, in Kauppinen series, ${ }^{14}$ the largest number of cases was seen in the 6th decade. This could probably be related to the regional variation in health care seeking patterns. Of the various types of cutaneous ADR, maculopapular rash was the commonest seen in children in this study, as was documented previously. ${ }^{15}$ Since the general prevalence of NSAID hypersensitivity in the pediatric age-group is low, it would be a significant advantage if diagnostic challenge protocols and diagnostic criteria could be developed and agreed on in an 
international consensus, thus facilitating the comparison and integration of developed data.

\section{CONCLUSION}

Cutaneous eruptions are a commonly reported adverse drug reactions associated with the use of the NSAIDs. Cutaneous adverse drug reactions in the pediatric population have a significant impact on patients' current and future care modalities. The purpose of this study was to understand a reasonable approach to recognition and initial management of cutaneous adverse drug reactions in children. Since all the 20 patients with NSAIDsassociated ADRs experienced resolution of their symptoms after discontinuing the offending agent, we propose that this clinical correlation alone might be sufficient to confirm the diagnosis of fixed drug eruptions.

\section{ACKNOWLEDGEMENTS}

The author(s) deeply acknowledge the faculty members of the Department of Dermatology and Department of Pediatric Medicine, for prompt diagnosis and treatment of the affected children in this study.

Funding: No funding sources

Conflict of interest: None declared

Ethical approval: The study was approved by the Institution Human Ethics Committee

\section{REFERENCES}

1. Walling HW, Swick BL. Cutaneous fixed drug eruption to fluconazole. J Drugs Dermatol. 2010;9:1025-8.

2. Breathnach SM. Drug reactions. In: Burns T, Breathnach S, Cox N, Griffiths C, editors. Rook's Textbook of Dermatology. $8^{\text {th }}$ ed. Oxford: Blackwell Science; 2010: 28-177.

3. Brocq L. Éruptionerythemato-pigmentée fixe due al'antipyrine. Ann Dermatol Venereol. 1894;5:30813.

4. Kidon MI, Kang LW, Chin CW, Hoon LS, and Hugo VB. Nonsteroidal Anti-Inflammatory Drug
Hypersensitivity in Preschool Children Allergy, Asthma, and Clinical Immunology. 2007;3:114-22

5. Bigby M, Jick S, Jick H, Arndt K. Drug-induced cutaneous reactions: a report from the Boston Collaborative Drug Surveillance Program on 15,438 consecutive inpatients, 1975 to 1982 . JAMA. 1986;256:3358-63.

6. Mani MZ, Mathew M. A study of 218 drug eruptions. Indian Journal of Dermatology Venereology \& Leprology. 1983;49:109-17.

7. Geneva: World Health Organization; World Health Organization. Technical Report Series No. 498. International drug monitoring: the role of national centers. 1972. Available at: www.whoumc.org/graphics/9277.pdf.

8. Naranjo CA, Busto U, Sellers EM, Sandor P, Ruiz I, Roberts EA, et al. A method for estimating the probability of adverse drug reactions. Clin Pharmacol Ther. 1981;30(2):239-45.

9. Hartwig SC, Siegel J, Schneider PJ. Preventability and severity assessment in reporting adverse drug reactions. Am J Hosp Pharm. 1992;49(9):2229-32.

10. Schumock GT, Thornton JP. Focusing on the preventability of adverse drug reactions. Hosp Pharm. 1992;27:538.

11. Priyadharsini, R., Surendiran, A., Adithan, C., Sreenivasan, S., \& Sahoo, F. K. A study of adverse drug reactions in pediatric patients. Journal of pharmacology \& pharmacotherapeutics. 2011;2(4):277.

12. Mehta TK, Marquis L, Shelty JN. A study of 70 cases of drug eruptions. Indian Journal of Dermatology Venereology \& Leprology. 1971;37:15 .

13. Kauppinen K. Cutaneous reaction of drugs. Acta Derm Venereol. 1972;52(Suppl 68):1-89.

14. Alanko K, Stubb S, Kauppinen K. Cutaneous drug reactions: clinical types and causative agents. Acta Derm Venereol (Stockh). 1989;69:223-6.

15. Berde CB, Sethna NF. Analgesics for the Treatment of Pain in Children. N Engl J Med. 2002;347:1094103.

doi:10.5455/2319-2003.ijbcp20140228

Cite this article as: Santra R, Mondal S, Raychaudhuri P. Nonsteroidal anti-inflammatory drug (NSAID) associated fixed drug eruption (FDE) in children attending dermatology OPD of a tertiary care hospital of Eastern India: a cross-sectional observational study. Int J Basic Clin Pharmacol 2014;3:211-4. 